\title{
Concepts and Derivatives of Web Services
}

\author{
Atieh Khanjani and Wan Nurhayati Wan Ab. Rahman \\ Faculty of Computer Science and Information TechnologyUniversitiPutra Malaysia 43400 UPMSerdang, Se- \\ langor, Malaysia
}

\begin{abstract}
Since the web services are growing rapidly in cloud, service consumers and providers are looking for a means to find better services that satisfy both parties. From both user's and developer's perspectives, discovering functional and non-functional characteristics of a web service is essentially considerable. Due to overcoming the above issues many research have been published to improve the usage of web services to satisfy customers. The paper reviews the literature of web services with respect to quality of service (QoS) or nonfunctional properties, to acquire better understanding of concepts and issues related to QoS web service selecting and discovering processes.
\end{abstract}

Keywords - web service, QoS, standards, UDDI, WSDL, SOAP, non-functional properties

\section{Introduction}

As today, businesses are willing to make their solutions on the web services technology, the need to have more and more acceptable and reliable one seems clear and this may not be able except improving the quality of web services through adding non-functional properties and applying high quality standards to satisfy both customer and marketplace vendors.Web services are self-describing and modular components that can be advertised, published, located and discovered through the Internet by using such a standards and protocols as Simple Object Access Protocol (SOAP), Universal Description Discovery and Integration (UDDI) and Web Service Description Language (WSDL)[1]. Access to services over the internet has relied on the interaction between a web server and a browser through using the HTTP protocol. The programmatic way to access to the services over the internet, called web services [2].

An internet (web) application can request several services such as payment services which can invoke an authentication services. Such a system is identified as a composite web services which is established statistically or dynamically. Dynamic web service composition needs service consumer to discover service provider to cope with the given functional and non-functional requirements[3].The non-functional requirements are also known as Quality of Service (QoS) requirements. Traditional web sites fulfill all components required to perform user transactions such as user interface, business logic, and access to the data base while web services sites, give users the opportunity to access to some or all of these services through a user interface- programs that provide these services over the web[2]. However, with emerging and increasing number of web services on the Internet web investigating quality of web services is becoming more and more considerable. Different web service QoS characteristics can be categorized as:

- $\quad$ User-independent or certain properties such as price and popularity that advertised by provider side;

- User-dependentor uncertain QoS propertiessuch as failure, response time and throughput which is related to the users and can be influenced by the Internet connection and user environments[4].

The following part of the paper consists of history of web services discussed in section 2, QoS requirements for web services stated in section 3, web service life cycle in section 4, web service versus web application presented in section5, two perspectives of web services for QoS in section 6 and finally we have concluded our work.

\section{The History Of Web Services}

The term "web services" has been uttered for the first time by Bill Gates in 2000. However, web services did not come from only Microsoft and many firms are interested in building electronic marketplaces. Electronic Data Interchange (EDI) is the first effort for making a standard for e-businesses came on the scene 25 years ago. On that time, there have been a lot of efforts in the world for connecting business logic over the Internet such as Common Object Request Broker Architecture (CORBA), Java Remote Method Invocation (RMI) and Unix Remote Procedure Call (RPC). However, these standardsfailed to earnimportant market share or adequate force to succeed. EDI and CORBA (an OMG standard which enables software components interact with each other through multiple languages and platform) were difficult to implement because of their complexity,cost, lack of flexibility and industrysupport.

Previously, getting agreement from vendors for a network service transport protocol were impossible while with emergingweb this problem solved because the web representedan academic decision throughintro- 
ducing the lower level transports (TCP/IP\& HTTP) for standardized communication.During that time the web went in its mainstream in 1994, TCP/IP was a mature standard. Http, in 1997 is known as a universal business standard. With emerging HTTP and TCP/IP, the main work was regarding to messaging, data encapsulation and cooperation between vendors.

XML invention makes the way proper for web services. XML officially became a platformindependent and proper standard for deployment in applications in 1998. In December 1999, while the electronic marketplace was the hot topic. The Microsoft through having a meeting with IBM and other companies represented the SOAP 1.0 and its definition for being a standardized protocol based on XML for messagepassing.

By year 2000, while the SOAP was gaining wider acceptance, the IBM and Microsoft merged their protocol proposals regarding contributed network accessible service specification language and WSDL and SOAP respectively and in the autumn they announced the merged WSDL. With SOAP and WSDL, companies could create and describe their web services. But what about the advertisement and locate of the services provided by service provider. However in March 2000, IBM, Microsoft, and Ariba started working on the way to place the services that was Universal Description, Discovery, and Integration (UDDI).UDDI 1.0 was introduced in September 2000.At the end of 2000 with working these three standards together (SOAP, WSDL, and UDDI), the defacto standards to develop web services had created[5].

\section{QoSRequirementsfor Web Services}

QoS that is a set of non-functional properties is an importantparameter to distinguish the web services. QoS is also one of the factors to enhance the adoption rate of web services and impact the quality of web services as well[6]. The need to consider QoS properties for web services is because of attracting more users and improving the quality of WS.In fact in terms of providing a service, when a service provider only considers to functional requirement, the provided services will not satisfy users at runtime.Moreover since WSDL only describes functional requirements of the system and is not able to describe non-functional properties, we need to consider QoS to satisfy customer[7].Some of the most important QoS properties are presenting as follows:

- Accuracy: represents the error rate which is produced by the service.

- Scalability: represents ability of web services to consistently fulfill the requests although there are numbers of request's volume.

- Capability: represents the numbers of requests handled by service [8].

- Performance:In [9]the authors stated that performance can be measured by throughput and latency. Good performance comes from lower latency and higher throughput. Besides, performance can be determined by the response time to make sure that the maximum time needed to fulfill a service request has been gotten.

- Throughput: number of invokes that is served at a specified time period.

- Latency: round-trip time tosend a request and receive the answer.

- Reliability: measures how the quality of web services performs through a specific time and network conditions. WS-Reliability guarantees that a message is delivered exactly one time [10].

- Availability:determines that the web services are ready for immediate use and a service is available or not. If the value is high it shows the service is ready to use while if it is small it means that the service can be used only in particular time[11].

- Flexibility: represents how a service can function correctly when the input is invalid, incomplete or conflict.

- Exception handling: represents how the service handles the exceptions (special cases or unanticipated possibilities) [6].

- Integrity:shows the capability of web services to maintain the correctness of the resource interaction.

- Regularity: is a quality factor that follows the rules and laws, compliance with standards and service level agreement.

- Stability: is the services's attributes modification rate [8].

- Cost: represents how to measure the cost of service requesting usually based on the amount of request or the volume of data.

- Completeness: represents how to measure the difference between the specified and implemented set of features.

- Security:is the quality factor which can be measured by authenticating, encrypting and access control to messaging.

- Accessibility: represents capability to serve a request. Or probability that measuresrecognition of the success rate of service introduced at a point of time. Higher accessibility can be achieved by higher scalability of the system.

- Dependability: represents how to deliver a service that can justifiably be trusted[1].

- Compliance: is a property that determines whether the service is compatible with standards or not. 
- Trustiness: depends on the experience of the users which use the system. It alsoevaluates the reliability of user reports.

\section{Web Service Life Cycle}

In the fundamental web service life cycle process, there is three parts: service provider, service customer and UDDI registry; however in some cases there is no UDDI interfering, so the provider and user can communicate directly to each other[12],[13]. Figure1 indicates the process of web services. The service providers publish their service to UDDI registry through the IP address or an identification key. The client asks for the services from UDDI, the registry then checks in the data base and returns back the service to the user. The client may invoke directly the service from provider as well. But the problem is that in this model the user does not know about the quality of web services. To solve such a problem, some researchers proposed models regarding considering QoS properties while finding a web service [12],[14],[15],[16].

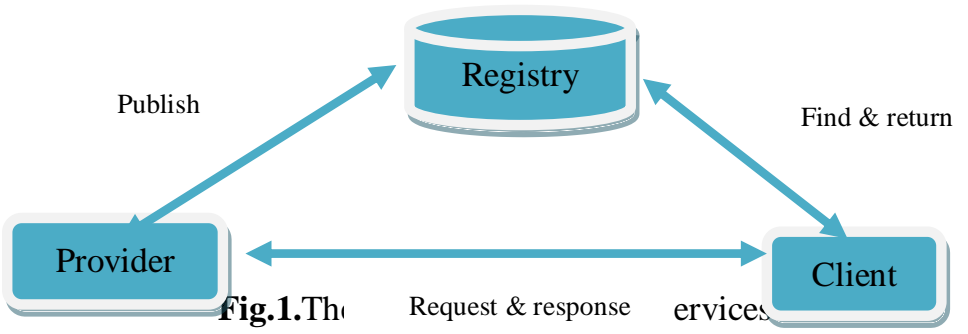

Ran [6] proposed a web service registration and discovery model that applied a certifier to verify nonfunctional properties (QoS) of web services. Another research [17] suggested a web service broker to interaction between client, provider and UDDI. In some researches, they added the QoS to the registry - UDDI [18]or extend the WSDL file to support the non-functional properties as well [19]. In [20], they proposed an approach for service selection based on authenticity of QoS and user confidence. Regarding to discovering web services another research performed by using AI methods (Neural Network) to find a best solution [21]. The same authors in another article added a QoS manager to web service selection which enables users to control the process of service discovery [22]. In [23], they proposed a web service selection model based on the QoS properties (WSSM-Q) to provide QoS support for publishing and searching the web services considering a rank for the services to be selected with high quality.The authors in [24] completed the previous work by adding a threshold value for QoS properties and practically examined the model by creating insurance application. An efficient discovery model based on QoS and meta data presented in [25]. In another paper they proposed six dimensional (agreed, expected, transmitted, statistic, delivered and perceived) QoS framework to access quality of web services[26]. A work on QoS ontology for service discovery to solve the problems of syntax service discovery has been done when there are a large number of developers and user exist [27]. Similar works related to the QoS ontology for web service selection performed by [28], [29], and [8]. To evaluate web services and access quality of web services also a lot of researches has been done using common criteria [30], choice-making method [31], unified model [32], agents [33],[34],[35]and adaptive algorithm [36]to predict web service QoS.Table 1 indicates the comparison between fundamental models of web services life cycle process which consider QoS.

Table 1.Comparison between QoS models

\begin{tabular}{|c|c|c|c|c|}
\hline Models & Author & $\begin{array}{c}\text { Yea } \\
\mathbf{r}\end{array}$ & Benefits & Limitations \\
\hline Broker [35] & $\begin{array}{l}\text { Rajendran } \\
\text { and Balasu- } \\
\text { bramanie }\end{array}$ & 2010 & $\begin{array}{l}\text { Optimal and agent based dynamic service discov- } \\
\text { ery architecture, add certifier }\end{array}$ & User perspective \\
\hline Certifier [6] & Ran & 2003 & Used certifier to rank & User perspective \\
\hline Extend UDDI [8] & Yin et al. & 2010 & $\begin{array}{l}\text { QoS framework with ontology, OWL-S/UDDI } \\
\text { translator (both numerical \& semantic) }\end{array}$ & $\begin{array}{l}\text { Did not mention to } \\
\text { certification and } \\
\text { ranking of QoS }\end{array}$ \\
\hline WSSM-Q [23] & Liu et al. & 2009 & Ranking & User perspective \\
\hline QoS Threshold [34] & $\begin{array}{l}\text { Raj and } \\
\text { Sasipraba }\end{array}$ & 2010 & Completed [35] with threshold & User perspective \\
\hline Six-dimensional [26] & Nanet al. & 2008 & $\begin{array}{l}\text { Six-dimensional QoSW framework to describe in } \\
\text { SLA, monitor and assess. Both user and provider } \\
\text { side. }\end{array}$ & Not evaluated. \\
\hline Q-WSDL [19] & D'Ambrogio & 2006 & $\begin{array}{l}\text { Extension of WSDL by introducing the metamo- } \\
\text { del to enable QoS for WSDL when registering or } \\
\text { establish SLA }\end{array}$ & Not evaluated \\
\hline $\begin{array}{l}\text { AI methods (Neural } \\
\text { Network) [21] }\end{array}$ & $\begin{array}{l}\text { Al-Masri and } \\
\text { Mahmoud }\end{array}$ & 2009 & $\begin{array}{l}\text { Apply ANN nodes and the performance is } 95 \% \\
\text { success to find the desired web service }\end{array}$ & $\begin{array}{l}\text { User perspec- } \\
\text { tive/discovery phase }\end{array}$ \\
\hline
\end{tabular}




\begin{tabular}{|c|l|l|l|l|}
\hline $\begin{array}{c}\text { WS Relevancy Func- } \\
\text { tion (WsRF) \& WS- } \\
\text { QoS Manager [22] }\end{array}$ & $\begin{array}{l}\text { Al-Masri and } \\
\text { Mahmoud }\end{array}$ & 2007 & $\begin{array}{l}\text { Measuring therelevancy ranking of a Web service } \\
\text { based on QoS\& user preferences. }\end{array}$ & $\begin{array}{l}\text { User perspec- } \\
\text { tive/discovery phase }\end{array}$ \\
\hline Broker [17] & Tian & 2003 & $\begin{array}{l}\text { Agent to support \& manage QoS keeps the in- } \\
\text { formation up to date. Remove the expired offer } \\
\text { from the catch. Extension for WSDL file to } \\
\text { support QoS }\end{array}$ & $\begin{array}{l}\text { There is no ranking } \\
\text { and weighting for the } \\
\text { QoS selection. }\end{array}$ \\
\hline
\end{tabular}

The result of the comparison illustrates that most of the researches currently are focusion user perspective, discovery phase and description phase while there is still a huge lack of work on provider perspective and development phase.

\section{Web Services Versus Web Applications}

Web applications are more widely than web services, a web service can be a part of web application but web application may not be a web service.Moreover, when you look at a web page you have to use a web browser (Internet Explorer or Firefox) to query a web server for viewing the web page while in web services because of using SOAP technology, you are able to use your application to query a server and run a program.

Consider thatsuch a kind of things in web pages standards or HTML is impossible. An example for better understanding of the differences between web pages and web services can be the online banking or hotel booking services.In online banking service, if we apply web pages we have to access to the bank account (e.g. to transfer money), bill paying and a page related to manage the credit cards. Although these three applications are separate, it means while paying the bills cannot view the account balance and this is because they reside on different platform and run by different servers. But when we are using web services through SOAP protocol this problem will be solved because SOAP is not rely on the platform and language[37]. In the other word a web service is an online service available through the internet via HTTP for interaction with the other programs.

Against web pages that user should interact through a GUI, in web services there is no GUI for interaction. Because it uses XML document and need a client-side parser. As a matter of fact, the web services are mostly like application components that can be used by another application. Furthermore the main goal of service discovery is quite different from web searches. Usually users search for the web pages to find a source or specific interested topic while consumer search for the web services to find an interesting domain to integrate to their systems. Moreover client not only looks for the web services to meet the requirement but the founded services need to consider a degree of QoS as well [38].

\section{QoSWeb Services Perspectives}

There are two perspectives for considering QoS in web services: users and providers. Both perspectives are trying to enhance the quality of web services by considering QoS as one of the essential factors for improving the quality of web services. From the user side perspective, it is important to attend the level of satisfaction and consistency of QoS parameters with the user's desire. However there are some QoS parameters that should be consider through user preference with filling up the forms and voting process at the execution time of the web services to show how much they were satisfy from the web services and with which specific QoS because some of the QoS parameters are domain-relevant and should be consider only to that specific services. Reputation is one of the most famous QoS attribute related to the user side perspective. Furthermore enabling clients for customizing their own discovery process is one of the challenging issues in recent research works(Al-Masri and Mahmoud, 2007). From the other side provider perspective is to consider QoS aspects of web services at the beginning stage and to publish and advertise web services to the registries (like UDDI). In this perspective provider tries to improve the quality of their web services by incorporating the QoS in the design stage (like predicting QoS).Most of the recent researchers concentrated on client side to incorporate the user preference for selecting and discovering the best web services. Table 2 shows the QoS attributes for two perspectives of WS which is the user and provider. However, as it is clear some attributes can be affected by both perspectives.

Table 2.The QoS attributes from web service perspectives

\begin{tabular}{c|c}
\hline \hline User perspective & Provider perspective \\
\hline Reputation & Time-to-market \\
\hline Availability & Availability \\
\hline Performance & Performance \\
\hline Accessibility & Failure rate \\
\hline Speedy & Compliance \\
\hline Scalability & Conceptual integrity \\
\hline Privacy & Security \\
\hline Reliability & Reliability \\
\hline \hline
\end{tabular}


Table 2 shows the most important and common attributes of QoS among two perspectives. As it is clear from the table some of these attributes can be consider only from one side such as reputation which usually consider only from user perspective and some of them should be consider for both perspective such as reliability.

\section{Conclusion}

Web services came to solve many problems of the life. Each service is to solve one or more problem. However, what if there are two or more similar web services available for one problem. Therefore, the QoS of the web service is an important consideration. Thus, to have a successful web services that satisfy both consumers and providers, the consideration of QoS issues seems critical and vital. Since QoS for web services is still a new topic there been extensive researches in finding a suitable and viable wayhow to improve the quality of web services. The paper reviews the concepts and fundamental aspects of QoS web services to acquire better understanding how the web services works under QoS parameters. The paper shows how the process of considering QoS in web service life cycle is improving by the comparison between four fundamental models. In addition, the paper has highlighted the lacked of QoS parameters consideration especially at the provider side.

\section{References}

[1] Kritikos K and Plexousakis D, Requirements for QoS-Based Web Service Description and Discovery. Services Com puting, IEEE Transactions on 2, 2009, 320-37.

[2] Menasce DA, QoS issues in Web services. Internet Computing, IEEE 6, 2002, 72-5.

[3] Menasce DA, Composing Web Services: A QoS View. Internet Computing, IEEE 8, 2004, 88-90.

[4] Zibin Z, Yilei Z, and Lyu MR, Distributed QoS Evaluation for Real-World Web Services. In Web Ser IEEE International Conference on. (ed.), Vol., 2010, pp. 83-90.

vices (ICWS)

[5] Levitt J, From EDI To XML And UDDI. In A Brief History Of Web Services. (ed.), Vol., 2001, pp.

[6] Ran S, A model for web services discovery with QoS. SIGecom Exch., 4, 2003, 1-10.

[7] Yan-ping Chen Z-zL, Qin-xue Jin, and Chuang Wang, Study on QoS Driven Web Services Composi tion. Springer-Verlag Berlin Heidelberg, 3841, 2006, 702-7.

[8] Yin B, Yang H, Fu P, Gu L, and Liu M, A framework and QoS based web services discovery. In Soft ware Engineer ing and Service Sciences (ICSESS), IEEE International Conference on. (ed.), Vol., $\quad 2010$, pp. 755-8,

[9] W.N.WanAbRahman and F.Meziane, Challenges to Describe QoS Requirements for Web Services Quality Prediction to Sup port Web Services Interoperability in Electronic Commerce. Communications of the IBIMA, 4, 2008, 50-8.

[10] Yu WD, Radhakrishna RB, Pingali S, and Kolluri V, Modeling the Measurements of QoS Require ments in Web Service Systems. Simulation 83, 2007, 75-91.

[11] Farsandaj K and Ding C, Scatter/Gather browsing of web service QoS data. Future Generation Com puter Systems 28, 2012, 1145-54.

[12] Gang Y, Chanle W, Jun Y, and Shi C, A QoS-Aware Model for Web Services Discovery. In Education Technology and Computer Science, 2009.ETCS '09.First International Workshop on. (ed.), Vol. 3, 2009, pp. 740-4,

[13] Minder Chen ANKCaBBMS, The Implications and Impacts of Web Services to Electronic Commerce Research and Practices. J. Electron. Commerce Res., 4, 2003, 128-39s.

[14] IIavarasanEgambaram GV, S. PrasathSivasubramanian, QOS BASED WEB SERVICE SELECTION. 2009, pp. CiteSeer, Pondicherry.

[15] Qi X, Kaigui W, and Jie X, QoS Driven Web Services Evolution. In Complex, Intelligent and Software Intensive Sys tems (CISIS), 2011 International Conference on. (ed.), Vol., 2011, pp. 329-34,

[16] S. Maheswari GRK, QoS Based Efficient Web Service Selection. European Journal of Scientific Re search 66, 2011, 428-40.

[17] Tian M, Gramm A, Naumowicz T, Ritter H, and Freie JS, A concept for QoS integration in Web ser vices. In Web Information Systems Engineering Workshops, 2003.Proceedings.Fourth International Conference on. (ed.), Vol., 2003, pp. 149-55,

[18] Xiaopeng D and Chunxiao X, A QoS-oriented Optimization Model for Web Service Group. In Com puter and Information Science, ICIS 2009.Eighth IEEE/ACIS International Conference on. (ed.), Vol., $\quad$ 2009, pp. 903-9,

[19] D'Ambrogio A, A Model-driven WSDL Extension for Describing the QoSofWeb Services. In Web Services, ICWS '06.Inter national Conference on. (ed.), Vol., 2006, pp. 789-96,

[20] Yuan Yu q and Hu Xiao h, A Web Service Selection Approach Based on the Authenticity of QoS Data and the Confi dence of Users. In Computer Network and Multimedia Technology, CNMT 2009.Inter national Symposium on. (ed.), Vol., 2009, pp. 1-5,

[21] Al-Masri E and Mahmoud QH, Discovering the best web service: A neural network-based solution. In Systems, Man and Cybernetics, SMC 2009.IEEE International Conference on. (ed.), Vol., 2009a, pp. 4250-5,

[22] Al-Masri E and Mahmoud QH, Discovering the best web service. In Proceedings of the 16th interna tional conference on World Wide Web. (ed.), Vol., 2007, pp. 1257-8, ACM, Banff, Alberta, Canada. 